\title{
45. Demonstration of Xenotropic Murine Leukemia Virus-related Sequences in Various Rodent Species
}

\author{
By Noriyuki Sagata,*) Kazuo Moriwaki,**) and Yoji IkaWa*) \\ (Communicated by Toshio Kurokawa, M. J. A., June 15, 1982)
}

Introduction. All vertebrates appear to carry in their genomes endogenous retroviruses which are transmitted vertically. ${ }^{1)}$ In mice, endogenous type $\mathrm{C}$ retroviruses are divided into three classes on the basis of their host range; ecotropic, xenotropic and amphotropic murine leukemia viruses (MuLV).2),3) Mice of certain inbred strains (e.g., AKR) express high titers of endogenous ecotropic MuLV and develop thymic lymphomas. ${ }^{4}$ The ecotropic virus expression, however, is not sufficient for leukemia development,, ${ }^{\text {) }}$ and mink cell focusinducing (MCF) viruses, which are recombinants between ecotropic and xenotropic MuLV env sequences, appear to be a more proximate cause of leukemia.6) We report here, using a xenotropic MuLVspecific DNA sequence as a probe, that endogenous xenotropic or MCF virus-related sequences are carried by various rodents.

Materials and methods. Individual fresh liver specimens (from animals described later) were brought to DNA extraction. ${ }^{\tau)}$ The DNAs digested with a restriction enzyme Hinc II were subjected to agarose gel electrophoresis, transferred to a nitrocellulose filter ${ }^{8)}$ and hybridized with ${ }^{32} \mathrm{P}$-nick-translated probes under non-stringent conditions. ${ }^{9)}$ Filters were washed with concentrations of SSC $(6 \times$, $0.8 \times$ or $0.1 \times \mathrm{SSC}$ ) at $55^{\circ} \mathrm{C}$ to assess homology degree between probes and sample cellular DNAs.

Hybridization probes are shown in Fig. 1. The sequence specific for the Friend strain of spleen focus-forming virus (SFFV), designated as SSS (Fig. 1A), was $0.75 \mathrm{~Kb}$ in size and subcloned into the plasmid vector pBR322 from molecularly cloned SFFV DNA. ${ }^{10)}$ This probe had nucleic acid sequence homology with the env region of xenotropic MuLV.10),11) The second probe, long terminal repeat (LTR) of SFFV, was obtained as the internal Kpn I fragment from the two tandem LTRs of the cloned SFFV'10) (Fig. 1B) and could detect most of either type of LTR of MuLV. ${ }^{10)-12)}$ The third probe

*) Department of Viral Oncology, Cancer Institute, Toshima-ku, Tokyo 170, and Laboratory of Molecular Oncology, The Institute of Physical and Chemical Research, Wako, Saitama 351.

**) Department of Cytogenetics, National Institute of Genetics, Mishima 411. 
(A)

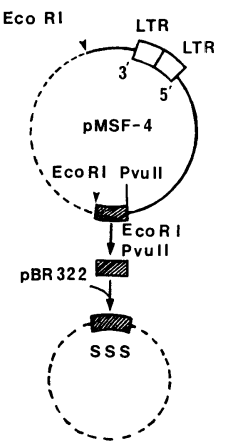

(B)

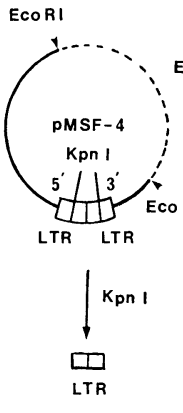

(C)

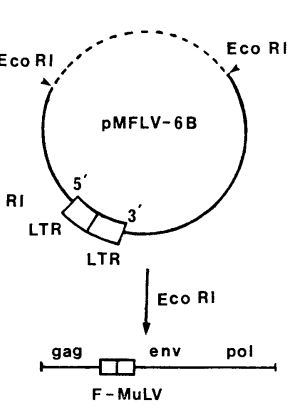

Fig. 1. Schematic representation of hybridization probes. Molecular cloning of unintegrated circular proviruses of both SFFV and F-MuLV has been described. ${ }^{10}$ Characterization and isolation of each hybridization probe are described in Materials and methods. A, SSS probe; B, LTR probe; C, F-MuLV probe.

was cloned, whole Friend MuLV genome, $9 \mathrm{~Kb}$ in size ${ }^{10)}$ (Fig. 1C) and having extensive sequence homology with genomes of both other ecotropic $\mathrm{MuLVs}^{13)-15)}$ and of xenotropic MuLVs except env region. ${ }^{6), 14)}$

Results and discussion. Hinc II-digested DNAs from various rodents were brought to a Southern blotting analysis using a xenotropic env-related sequence (SSS) and LTR of SFFV as the probes. Hinc II cuts most of the murine xenotropic provirus DNAs at the $5^{\prime}$ side of their env regions, giving rise to genomic DNA fragments tandemly composed of $e n v, 3^{\prime} \mathrm{LTR}$ and cellular flanking DNA. Hence, if one Hinc II-digested fragment from a given animal DNA has sequence homology with both SSS and LTR, the fragment is presumed to contain endogenously a xenotropic MuLV-related sequence.

Multiple xenotropic MuLV genes in Mus musculus subspecies. The SSS hybridized with multiple bands of both inbred (AKR, Fig. 2A) and feral (Mus musculus domesticus, Fig. 2B; M. m. molossinus, Fig. 2C) mouse DNAs. Many of these bands were also hybridizable with LTR, indicating the presence of multiple copies of endogenous xenotropic MuLV-like sequences. The number of copies of such xenotropic MuLV-like sequences in AKR mouse was estimated as 20-30 per genome, agreeing with data previously obtained by liquid hybridization. ${ }^{16)} M . m$. domesticus showed a hybridization pattern similar to that of AKR mouse (Fig. 2B), supporting the idea that inbred laboratory mice originated from the European mice. ${ }^{17), 18)} M$. m. molossinus, the Japanese feral mouse strain, showed quite a different pattern of hybridization with many more copies of xenotropic MuLV-related sequences (Fig. 2C). In all of these mice, however, both SSS and LTR remained hybridized with the multiple DNA 

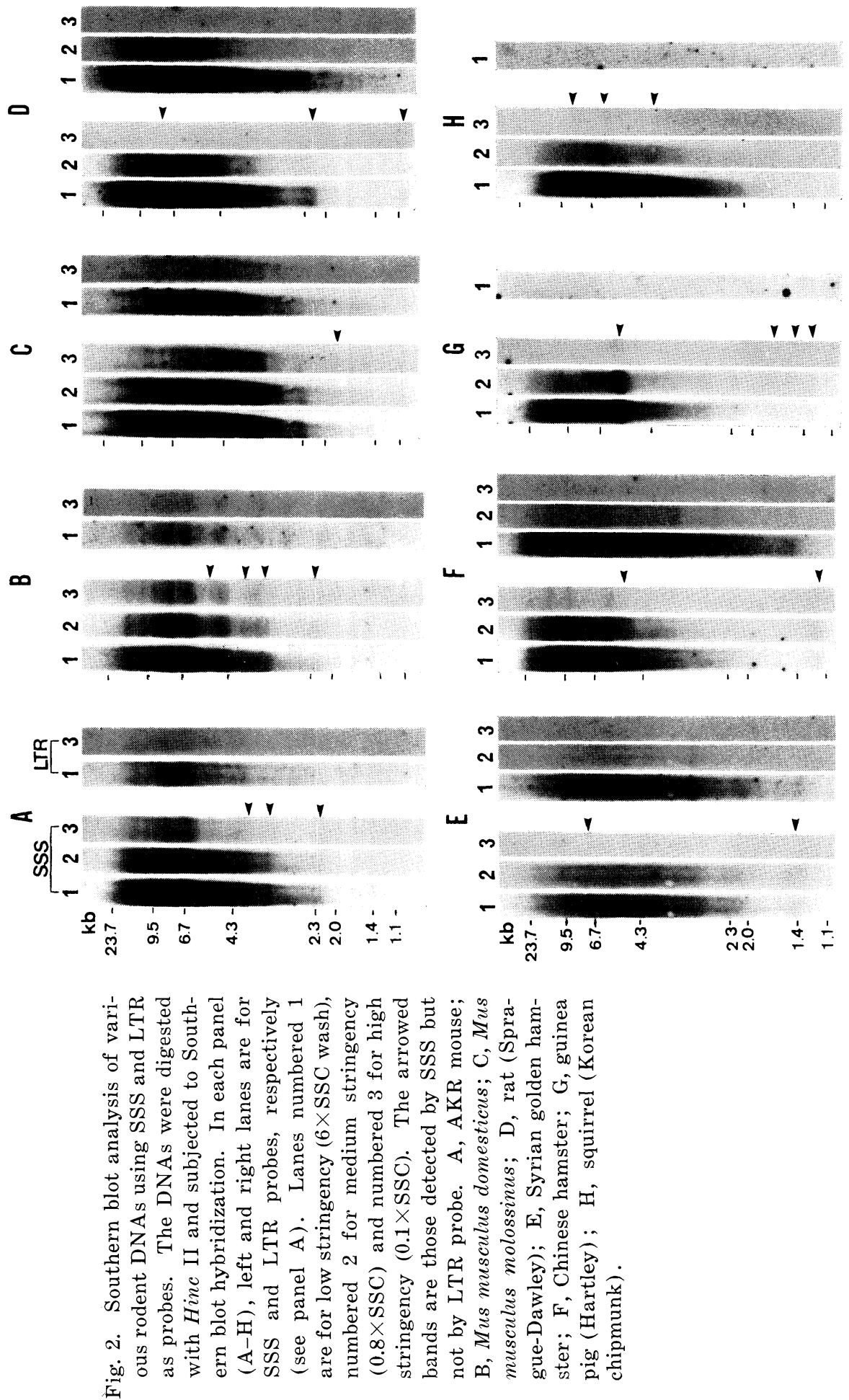
fragments even after stringent washing $(0.1 \times \mathrm{SSC})$ of the hybridized filters (no. 3 lanes). The Mus musculus subspecies thus appears to have closely related multiple copies of endogenous xenotropic $\mathrm{MuLV}$.

Xenotropic MuLV-related sequences in rats. In the rat, about. ten bands were detected by SSS probe above a high and smear background under non-stringent condition ( $6 \times \mathrm{SSC}$ wash) (Fig. 2D). With stringent washing, most of these bands and the smear background became quite faint, showing a sharp contrast with the mouse subspecies results. With LTR as a probe, several faint bands were detected over an unexpectedly high background, some appearing to correspond to the bands detected by SSS. A few fragments (arrowed in Fig. 2D) detected by SSS probe, however, did not hybridize to the LTR. These results indicate the presence in the rat DNA of xenotropic MuLV-related sequences more distant from the probes than those in the mouse subspecies.

Xenotropic MuLV-related sequences in hamsters. Hamsters (Syrian golden and Chinese) showed several bands which could hybridize to both SSS and LTR (Fig. 2, E, F). Those detected in the Syrian golden hamster DNA were rather faint and hardly detectable after stringent washing (Fig. 2E). In the Chinese hamster (Fig. $2 \mathrm{~F}$ ), however, three of the several bands also hybridizable with LTR had strong intensities and still remained hybridized to SSS even after stringent washing, as in the mouse. The LTR probe hybridized strongly to both hamster DNAs under non-stringent conditions (LTRprobed first lanes in Fig. 2, E, F). These bands and the smear high background, however, became scarcely detectable after stringent washing, suggesting slight relatedness to the MuLV LTR. These animals, as well as mouse and rat, had a few bands (arrowed in Fig. 2, E, F) which could hybridize to SSS but not to LTR.

Xenotropic $M u L V$-related sequences in other rodents. In guinea pig and squirrel (Korean chipmunk), SSS hybridized to one prominent and a few other faint fragments (Fig. 2, G, H). These bands, quite strong in the guinea pig, became progressively faint by washing with decreasing concentration of SSC, as in the rat and Syrian golden hamster. In contrast to mouse, rat and hamster, LTR hybridized neither to these fragments nor to any others even under a nonstringent condition $(6 \times \mathrm{SSC}$ wash).

SSS-related sequences in rodent species. In all rodents examined, one or sometimes more bands could be detected which hybridized to SSS but not to LTR. DNAs digested with Hinc II were then hybridized to DNA of cloned F-MuLV which contained MuLV gag, pol, ecotropic env but not xenotropic env genes. In many of the animals, extra and very strong bands were detected besides 


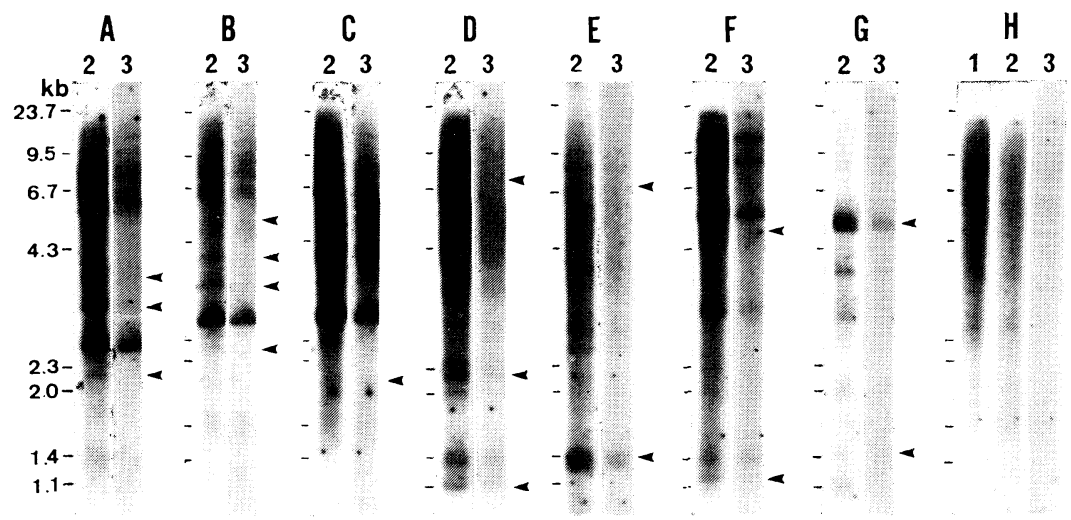

Fig. 3. Southern blot analysis of various rodent DNAs using F-MuLV DNA as a probe. The same experiments as in Fig. 2, except that the probe used was F-MuLV. The arrowed bands are those hybridized to SSS (but not to LTR, see Fig. 2) and to F-MuLV DNA.

the multiple SSS-probed bands. These are probably sequences homologous to gag or pol regions, the strong bands being the sum of internal fragments digested from the multiple copies of $\mathrm{MuLV}$ related endogenous sequences. A comparison of the bands arrowed in Fig. 2 with those in Fig. 3 revealed that almost all bands detected by SSS but not LTR in Fig. 2 could again hybridize to whole FMuLV DNA (for mouse, rat and two hamsters, Fig. 3, A-F), implying that these fragments are also derived from sequences related to xenotropic MuLV. In the guinea pig and squirrel, however, some of the bands detected by SSS but not LTR seemed not to hybridize to F-MuLV DNA (Fig. 3, G, H). These bands may suggest the presence of totally different endogenous viruses, ${ }^{10)}$ or of SSS-related sequences of cellular origin, ${ }^{20)}$ or a difference of divergence rate between LTR and non-LTR sequences.

Evolution of xenotropic MuLV genes in rodents. We have looked for xenotropic MuLV-related sequences endogenous in various rodents and found that all those examined possess them. Among non-murine rodents, the Chinese hamster appeared to have the most highly related endogenous sequences. The rat and Syrian golden hamster seemed to carry sequences distantly related, while the guinea pig and squirrel bore only inert vestiges of xenotropic MuLV, or totally unrelated endogenous viruses. These findings contrast with other reports describing the absence of xenotropic MuLV-related endogenous sequences in non-murine rodents, a result obtained using the standard liquid hybridization technique. ${ }^{21)}$ Our success may depend on the usage of the Southern transfer technique under nonstringent hybridization conditions using molecularly cloned probes. 
The presence of xenotropic MuLV-related sequences in all the rodents examined suggests that endogenous xenotropic $\mathrm{MuLV}$ has evolved from a common ancestral retrovirus carried in ancient rodents and amplified in the mouse and Chinese hamster. LTR sequences of the xenotropic MuLV might have evolved at a much higher rate than other viral genes because such sequences could hardly be detected in the evolutionally distant guinea pig and squirrel. The conservation of viral structural genes and the conversion of LTR sequences in retroviruses have been generally accepted.11),22)

Possible leukemogenecity of endogenous MuLV-related genes in rodents. In the $\mathrm{AKR}$ strain of inbred mice, the preleukemic and leukemic stages are associated with MCF virus. ${ }^{(6)}$ This leukemogenic virus is a recombinant between ecotropic $\mathrm{MuLV}$ and endogenous xenotropic MuLV env sequences. Similar situations may exist in naturally occurring leukemia in other rodents. Experiments to test this possibility are currently in progress in other vertebrates.

Acknowledgements. Mus musculus domesticus was the generous gift of Dr. M. L. Petras of Canada. We thank Dr. Haruo Sugano for his discussions during the work, Mr. N. Miyashita for the maintenance of animals, and Miss J. Kawamura for her excellent technical assistance. This work was partly supported by a Grant-inAid for Scientific Research from the Ministry of Education, Science and Culture, Japan, and a grant from the Tokushima Research Institute, Otsuka Pharmaceutical Co., Ltd., and by a research contract from the Science and Technology Agency of Japan.

\section{References}

1) Todaro, G. J., and Huebner, R. J.: Proc. Natl. Acad. Sci. U.S.A., 69, 1009 $(1972)$.

2) Levy, J. A.: Science, 182, 1151 (1973).

3) Hartley, J. W., and Rowe, W. P.: J. Virol., 19, 19 (1976).

4) Rowe, W. P.: Cancer Res., 33, 3061 (1973).

5) Lilly, F., Duran-Reynals, M., and Rowe, W. P.: J. Exp. Med., 151, 542 $(1980)$.

6) Chattopadhyay, S. K. et al.: Nature, 295, 25 (1982).

7) Onuma, M. et al.: Microbiol. Immunol. (in press).

8) Southern, E. M.: J. Mol. Biol., 98, 502 (1975).

9) Martin, M. A. et al.: J. Virol., 39, 835 (1981).

10) Sagata, N., and Ikawa, Y.: (submitted).

11) Chattopadhyay, S. K. et al.: Virology, 113, 465 (1981)

12) Yamamoto, Y. et al.: Proc. Natl. Acad. Sci. U.S.A., 78, 6893 (1981).

13) Bernstein, A. et al.: ibid., 76, 4455 (1979).

14) Bosselman, R. A. et al.: Virology, 102, 234 (1980).

15) Rands, E. et al.: ibid., 108, 445 (1981).

16) Chattopadhyay, S. K. et al.: Cold Spring Harbor Symp. Quant. Biol., 39, 1085 (1975).

17) Yonekawa, H. et al.: Japan. J. Genet., 55, 289 (1980)

18) Ferris, S. D., Sage, R., and Wilson, A. C.: Nature, 295, 163 (1982).

19) Besmer, P. et al.: J. Virol., 29, 1168 (1979).

20) Temin, H. M.: Annu. Rev. Genet., 8, 155 (1974).

21) Benveniste, R. E. et al.: J. Virol., 21, 849 (1977).

22) Cohen, M. et al.: ibid., 41, 801 (1982). 